УДК 539.37

С.В. Фирстов, С.В. Алышев, М.А. Мелькумов,

К.Е. Рюмкин, А.В. Шубин, Е.М. Дианов

Научный центр волоконной оптики РАН, Москва, Россия

ВОЛОКОННЫЕ СВЕТОВОДЫ, ЛЕГИРОВАННЫЕ

ВИСМУТОМ, - НОВАЯ АКТИВНАЯ СРЕДА ДЛЯ ЛАЗЕРОВ

И УСИЛИТЕЛЕЙ БЛИЖНЕГО ИК-ДИАПАЗОНА

Приведен обзор оптических свойств волоконных световодов, легированных висмутом. Представлены характеристики лучших по состоянию на данный момент лазеров и оптических усилителей созданных на основе волоконных световодов, легированных висмутом. Кратко обсуждены проблемы применения висмутовых активных сред.

Ключевые слова: висмут, волоконный световод, волоконный лазер, волоконный оптический усилитель.

S.V. Firstov, S.V. Alyshev, M.A. Melkumov, K.E. Rumkin, A.V. Shubin, E.M. Dianov

Fiber Optics Research Center of Russian Academy of Sciences, Moscow, Russian Federation

\title{
BISMUTH-DOPED OPTICAL FIBERS - A NEW ACTIVE MEDIUM FOR NIR LASERS AND OPTICAL AMPLIFIERS
}

An overview of the optical properties of bismuth-doped optical fibers is presented. The current state of the art in the creation of bismuth-doped fiber lasers and optical amplifiers is described. The problems accounted in practical use of the bismuth-doped fiber lasers and optical amplifiers are briefly discussed.

Keywords: bismuth, optical fiber, fiber laser, optical fiber amplifier.

\section{1. Введение}

Поиск и создание новых активных лазерных сред по-прежнему остается весьма актуальным из-за постоянной потребности в новых лазерах и оптических усилителях для многих областей применения, в частности для нового поколения волоконно-оптических систем связи. Современные коммерческие волоконно-оптические системы позволяют передавать информацию со скоростью до 10 Тбит/с по одному световоду, тогда как 
в экспериментальных системах скорость передачи информации достигает 100 Тбит/с. Это колоссальный успех в развитии волоконно-оптических систем связи и передачи информации, однако потребность в информации в развитых странах растет ежегодно на 30-40\%. Это означает, что через 10 лет возникнет потребность в передаче информации по одному волоконному световоду с петабитными скоростями. В литературе обсуждается ряд подходов к решению этой проблемы (см., например, работы $[1,2]$ и ссылки в них). Одним из них является расширение спектральной области для передачи информации. В современных высокоскоростных волоконно-оптических системах для этой цели используется узкая (80 нм) спектральная область 1530-1610 нм, определяемая полосой усиления эрбиевого волоконного усилителя. Но, как видно из рис. 1, область низких оптических потерь волоконных световодов на основе кварцевого стекла значительно шире. Так, спектральная область с оптическими потерями менее 0,4 дБ/км, которая могла бы быть использована для передачи информации, достигает 400 нм (1300-1700 нм).

Однако в настоящее время для спектральных областей 1300-1520 и 1610-1700 нм отсутствуют эффективные волоконные оптические усилители - обязательные элементы высокоскоростных волоконно-оптических систем связи и передачи информации.

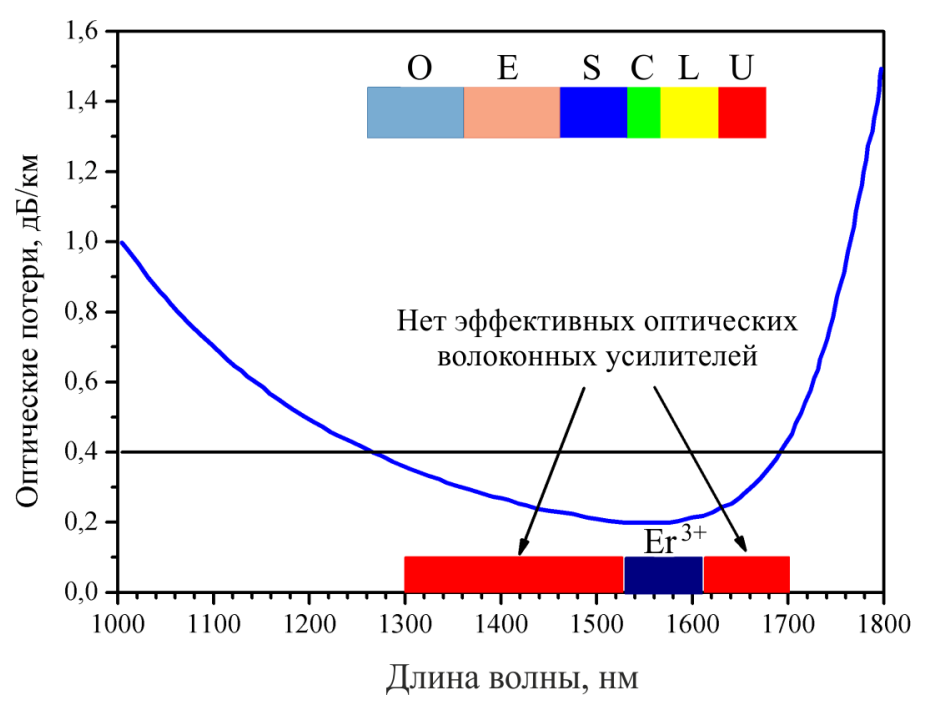

Рис. 1. Спектр оптических потерь волоконных световодов на основе кварцевого стекла и спектральная область работы эрбиевого волоконного усилителя $\left(\mathrm{Er}^{3+}\right)$, используемая в настоящее время для высокоскоростной передачи информации.

Также отмечены спектральные диапазоны O, E, S, C, L и U (терминология, используемая в телекоммуникационной литературе) 
Другой важной задачей, решение которой может потребоваться уже в ближайшие годы, является разработка пассивных оптических сетей с грубым (разреженным) спектральным уплотнением (Coarse WDM Next Generation Passive Optical Networks - CWDM NG - PONs). Эти системы предполагается использовать для доставки информации к конечному пользователю (сети доступа), что накладывает серьезные ограничения на их стоимость. Планируется, что данные системы будут работать в диапазоне длин волн 1271-1611 нм ${ }^{1}$. В этом случае потребуется оптический усилитель с полосой усиления 340 нм или 170 нм при разбиении полосы усиления на два диапазона для прямого и обратного трафика [3]. Пока создание любого из указанных усилителей является очень сложной задачей. Поэтому в данных системах предлагалось использовать различные схемы усиления на основе комбинирования полупроводниковых усилителей, эрбиевых волоконных усилителей, оптических параметрических и ВКР-усилителей [4]. Однако обеспечение оптического усиления по приемлемой для этих систем стоимости все еще встречает серьезные затруднения.

В этом контексте активные волоконные световоды, легированные висмутом, являются многообещающей средой, так как на данных типах световодов уже сейчас продемонстрировано оптическое усиление и лазерная генерация в диапазоне длин волн от 1146 до 1775 нм. Настоящая работа посвящена обзору оптических и усилительных свойств этих световодов.

\section{2. Оптические свойства волоконных световодов, легированных висмутом}

Люминесценция в ближнем инфракрасном диапазоне в стеклах, легированных висмутом, была обнаружена в 2001 г. [5]. Первый ИКлюминесцирующий легированный висмутом световод был изготовлен в 2005 г. [6, 7]. Всестороннее изучение оптических свойств данных активных сред показало, что характеристики формирующихся висмутовых активных центров (ВАЦ) в сильной степени определяются составом стекла сердцевины световодов. В результате многолетних исследований удалось выявить четыре класса ВАЦ в световодах на основе

${ }^{1}$ TU-T G. 694.2. Spectral grids for WDM aplications: CWDM wavelength grid Series G: Transmission systems and Media Digital Systems and Networks Transmission media characteristics Characteristics of optical component and subsystems. December 2003. 
кварцевого стекла, а именно: ВАЦ, ассоциированные с алюминием (ВАЦ-Аl); ВАЦ, ассоциированные с фосфором (ВАЦ-Р); ВАЦ, ассоциированные с кремнием (ВАЦ-Si), и ВАЦ, ассоциированные с германием (ВАЦ-Ge). Понятие «ассоциированные» означает, что соответствующие центры появляются в стекле, содержащем в значительных количествах данный элемент. Например, ВАЦ-Аl формируются в алюмосиликатных, алюмофосфоросиликатных, алюмогерманатных стеклах. Появление определенного класса ВАЦ устанавливалось по характерным полосам поглощения и люминесценции. Подробное обсуждение оптических свойств висмутовых активных центров в различных стеклянных матрицах приводится, например, в работах [8-11]. Здесь необходимо лишь отметить, что способность висмута образовывать различные ИК-люминесцирующие активные центры в зависимости от состава стекла делает его более универсальным по сравнению, например, с редкоземельными элементами. Другими словами, изменяя состав стекла сердцевины световода, мы можем сильно сдвигать его полосу люминесценции соответственно, и полосу оптического усиления - и тем самым добиваться оптического усиления и лазерной генерации в широком спектральном диапазоне.

На рис. 2 представлены характерные спектры потерь висмутовых световодов различного состава. Включение висмута в стекло сердцевины приводит к появлению в спектре потерь световода характерных полос поглощения в видимой и ближней ИК-области. Каждому световоду соответствуют определенные полосы поглощения. Кроме полос ВАЦ во всех световодах присутствует полоса поглощения ОН групп с максимумом на 1,38 мкм.

Рис. 3 демонстрирует спектры люминесценции и возбуждения люминесценции световодов, различающихся по химическому составу стекла сердцевины. Спектрами люминесценции определяется возможная рабочая спектральная область лазеров и усилителей, а спектрами возбуждения люминесценции - область излучения источников накачки. Видно, что спектральное положение полос люминесценции и возбуждения характерной люминесценции существенно зависит от химического состава стеклянной матрицы. Поэтому для получения лазерной генерации в широком спектральном диапазоне необходимо варьировать химический состав стекла сердцевины активного световода и источники накачки. 


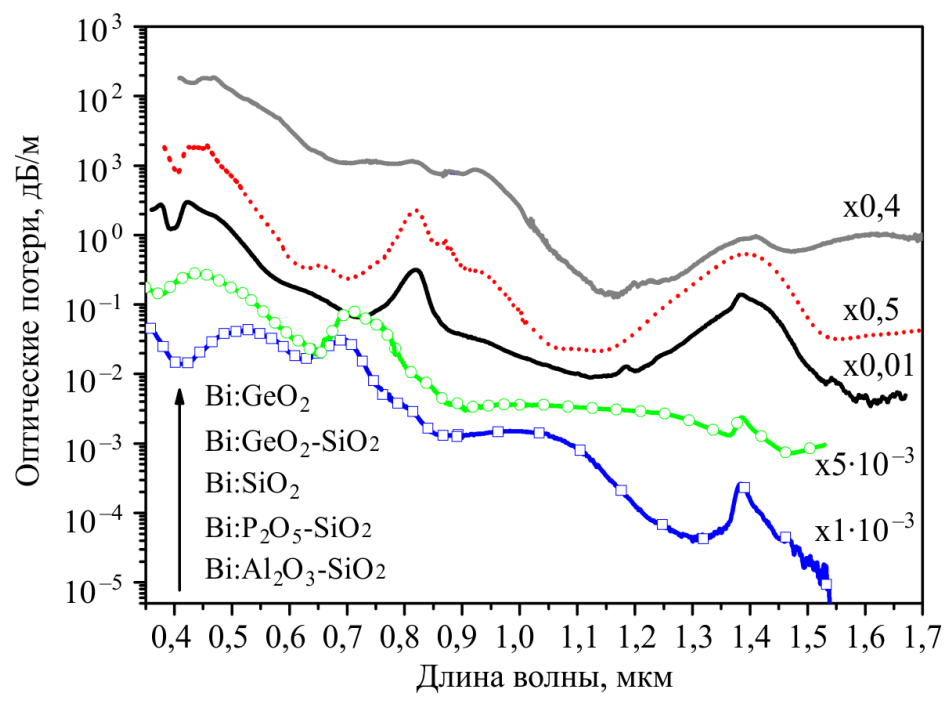

Рис. 2. Характерные оптические потери в легированных висмутом световодах с разными составами стекла сердцевины. Исходные спектры поглощения перекрывались друг с другом, поэтому для наглядности каждое значение соответствующего спектра потерь умножалось на коэффициент, приведенный справа от линии

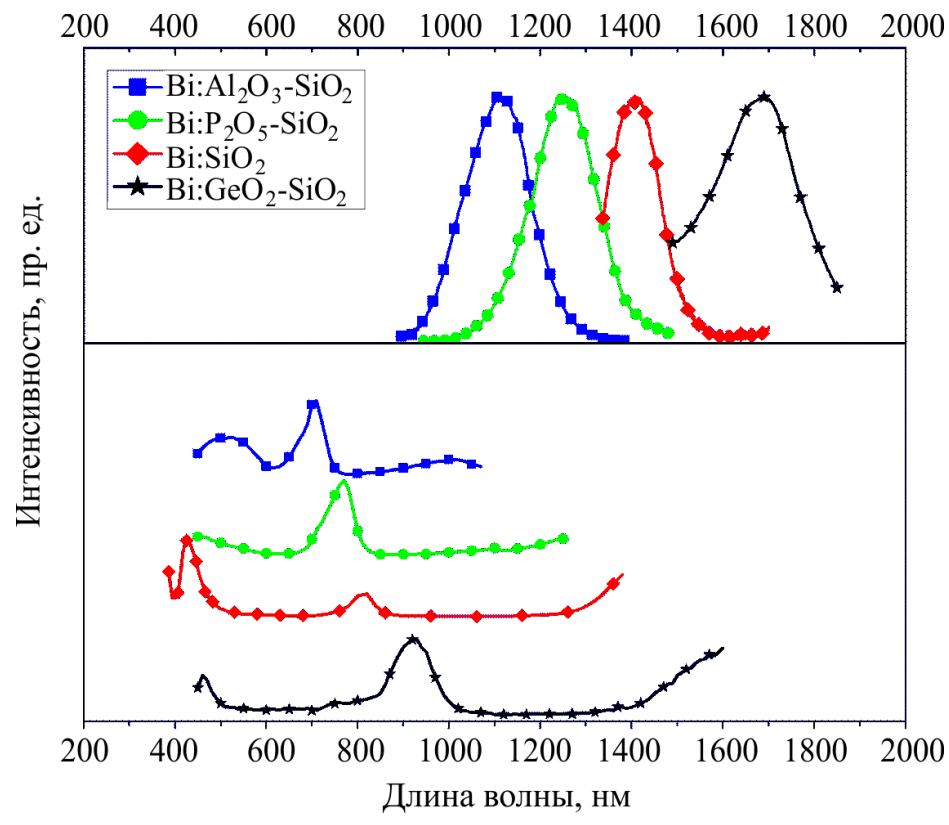

Рис. 3. Характерные спектры люминесценции (верхний график) и возбуждения люминесценции (нижний график) висмутовых световодов различного состава (световод из чистого кварцевого стекла, а также алюмосиликатный, фосфоросиликатный, германосиликатный световоды) 


\section{3. Лазеры}

Висмутовые волоконные лазеры создавались на основе световодов различного химического состава (см. предыдущий пункт), что позволяло получать лазерную генерацию в разных спектральных областях. Резонатор висмутовых лазеров формировался из активного световода и двух волоконных брэгговских решеток показателя преломления. Запись таких решеток осуществлялась УФ-облучением отрезков фоточувствительных световодов, которые затем приваривались к концам активного образца. Иногда для снижения внутрирезонаторных потерь брэгговские решетки записывались в сердцевине активного световода. Во всех экспериментах одна из решеток имела коэффициент отражения, близкий к $100 \%$ (глухое зеркало). Коэффициент отражения другой (выходной) решетки выбирался исходя из свойств активной среды (полупрозрачное зеркало). В некоторых схемах в качестве выходного зеркала служил торец оптического волокна. Длина волны накачки выбиралась в зависимости от конкретного типа световода. В качестве источников накачки использовались волоконные лазеры, работающие на эффекте вынужденного комбинационного рассеяния (ВКР-лазеры), Yb и $\mathrm{Er}-\mathrm{Yb}$ волоконные лазеры, твердотельный неодимовый лазер. Накачка вводилась в сердцевину активного световода.

Исторически первым лазером на основе активного волокна, легированного висмутом, был лазер на основе алюмосиликатного световода, т.е. лазер, использующий оптические переходы ВАЦ-Al [12]. На данном типе центров была достигнута лазерная генерация в диапазоне от 1146 до 1215 нм с низким значением пороговой мощности накачки ( 100 мВт). Позже было показано, что на данном типе световодов можно создавать лазеры с выходной мощностью 15 Вт $\left(\lambda_{\text {las }}=1160\right.$ нм $)$ [13] и КПД $\approx 28 \%$ [14]. Длина волны излучения накачки таких лазеров была в области 1060-1090 нм.

Висмутовые волоконные лазеры, излучающие в области длин волн от 1270 до 1360 нм, создавались на основе фосфоросиликатных световодов, легированных висмутом $[15,16]$. В настоящее время максимальная достигнутая мощность таких лазеров составляет 10,6 Вт (для $\lambda_{\text {las }}=1330$ нм), а КПД $\approx 40 \%$. В качестве источника накачки для таких лазеров используется ВКР-лазер, излучающий на длине волны 1230 нм.

До сих пор наилучшие результаты показывают висмутовые лазеры, работающие в области $\approx 1320-1540$ нм. В этом случае максимальная выходная мощность превышает 20 Вт (для $\lambda_{\text {las }}=1460$ нм). КПД ра- 
боты такого лазера составляет $60 \%$ [17]. Активной средой данного типа лазеров служит германосиликатный световод, легированный висмутом. Следует отметить, что лазерная генерация возникает на оптических переходах между энергетическими уровнями, принадлежащими ВАЦ- $\mathrm{Si}$, при возбуждении $\lambda_{\text {рump }}=1340$ нм ВКР-лазером. Однако в легированных висмутом германосиликатных световодах могут формироваться и ВАЦ-Ge, полоса люминесценции (усиления) которых расположена в области 1600-1800 нм.

Первые результаты показывают возможность лазерной генерации в области 1625-1775 нм на оптическом переходе между энергетическими уровнями ВАЦ-Ge с использованием в качестве накачки Er-Yb волоконного лазера, излучающего на длине волны 1568 нм. Максимальное значение КПД таких лазеров составляет $20 \%$ [18, 19]. Необходимо подчеркнуть, что генерация в данном диапазоне является новым результатом, и здесь еще остаются возможности дальнейшего улучшения характеристик.

Спектральные области лазерной генерации на различных типах волоконных световодов, легированных висмутом, представлены на рис. 4. На приведенном графике также отмечены спектральные диапазоны, ис-

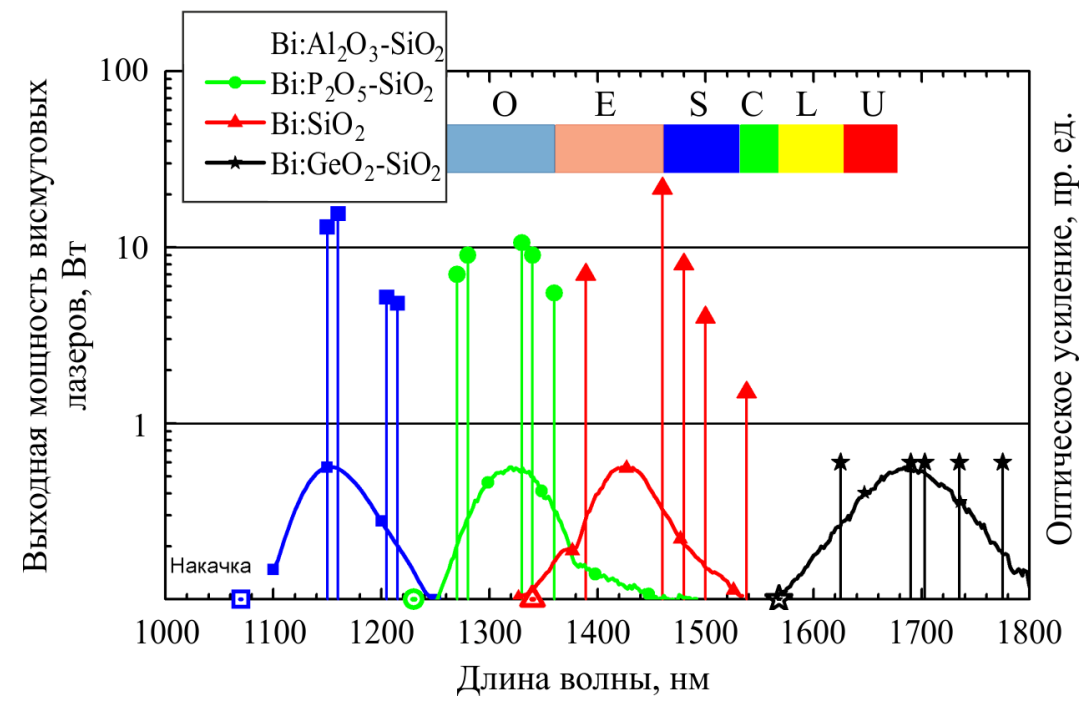

Рис. 4. Спектральный диапазон излучения лазеров на различных (по химическому составу стекла сердцевины) световодах, легированных висмутом. Положение вертикальных линий соответствует длинам волн, длины линий - мощностям лазеров. Также показаны нормированные спектры оптического усиления 
пользуемые в современных волоконных линиях связи, и спектры оптического усиления висмутовых световодов. Видно, что, комбинируя различные составы сердцевины, можно перекрыть лазерной генерацией широкий спектральный диапазон от 1100 до 1800 нм.

\section{4. Оптические усилители}

Приведенные выше результаты показывают, что световоды, легированные висмутом, могут служить активной средой для волоконных оптических усилителей, работающих в диапазоне 1100-1800 нм.

В настоящее время с использованием германосиликатных световодов разработан оптический усилитель для области 1430 нм с шириной полосы усиления по уровню 3 дБ $\Delta \lambda_{1 / 2}=36$ нм [20]. Рис. 5 показывает спектр усиления и спектральную зависимость шум-фактора данного усилителя. Пиковое усиление в 24 дБ достигалось при мощности накачки 65 мВт коммерчески доступным лазерным диодом с длиной волны излучения $\lambda_{\text {pump }}=1310$ нм. Шум-фактор в полосе $\Delta \lambda_{1 / 2}$ составлял 6 дБ. Максимальная эффективность усиления была равна 0,4 дБ/мВт. Это значение примерно на порядок меньше, чем типичные значения для эрбиевых волоконных усилителей, но на порядок выше, чем для типичного ВКР-усилителя, и в 2-4 раза выше, чем для тулиевого волоконного усилителя [20].

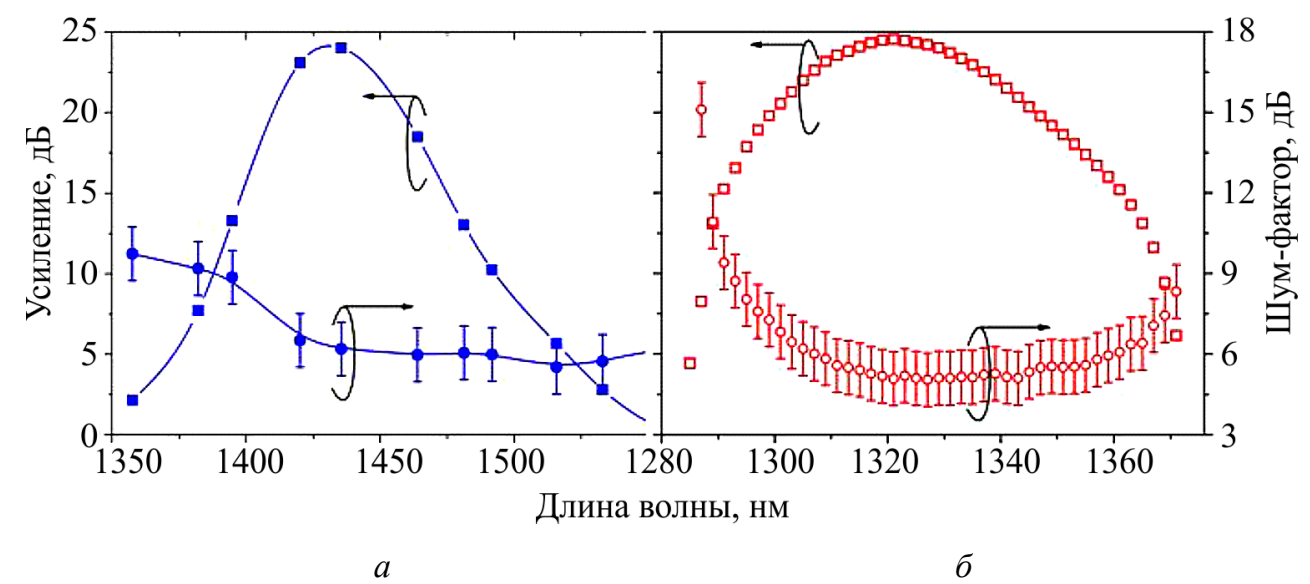

Рис. 5. Характеристики оптических усилителей на основе легированных висмутом световодов: $a$ - германосиликатного; $\sigma$ - фосфоросиликатного

Для области 1283-1372 нм также разработан оптический усилитель на основе фосфоросиликатного световода, легированного висму- 
том. Однако для достижения усиления 24,5 дБ в этом случае требовалась мощность в 6 раз выше (460 мВт от волоконного ВКР-лазера с $\lambda_{\text {pump }}=1230$ нм), чем для усилителя на 1430 нм [13]. Максимальная эффективность усиления составляла 0,09 дБ/мВт. Ширина полосы усиления $\Delta \lambda_{1 / 2}=37$ нм. Спектр оптического усиления и спектральная зависимость шум-фактора усилителя представлены на рис. 5,6 .

В случае алюмосиликатных световодов, легированных висмутом, КПД усилителя для области $\approx 1140-1215$ нм не превышает 0,01 дБ/мВт. Одной из причин низкого КПД является наличие в данных типах световодов поглощения из возбужденного состояния [21].

Усилительные свойства германосиликатных световодов в области 1600-1800 нм на данный момент находятся в состоянии исследования. Полученные результаты будут представлены в других работах.

\section{5. Проблемы применения висмутовых активных сред}

Несмотря на очевидный прогресс в области создания висмутовых лазеров и усилителей, существует ряд проблем, типичных при использовании новых лазерных материалов, которые пока не решены.

Отметим три ключевые проблемы:

1. Эффективная лазерная генерация на висмутовых волоконных световодах получена при низком общем содержании висмута (менее 0,02 ат. \%). Попытки увеличить концентрацию висмута приводят либо к возрастанию фоновых ненасыщаемых потерь [22], либо к формированию других активных центров с короткими временами жизни люминесценции [23].

2. КПД оптических висмутовых лазеров и усилителей ниже по сравнению с аналогичными устройствами с использованием световодов с редкоземельными ионами $\left(\mathrm{Er}^{3+}, \mathrm{Yb}^{3+}\right)$.

3. Отсутствие экспериментально подтвержденной модели висмутового активного центра создает определенные трудности при оптимизации химического состава стеклянной матрицы и технологии производства висмутовых волоконных световодов и, как следствие, не позволяет добиться оптимальных характеристик висмутовых лазеров и усилителей.

Трудности в использовании висмутовых сред в значительной степени связаны со своеобразием висмута как активного иона. $\mathrm{Bi}^{3+}$, входящий в состав исходных соединений при изготовлении волоконных световодов, быстро восстанавливается при высоких температурах (температурах плавле- 
ния стекла) с образованием разнообразных нанокластеров [24]. Проведение интенсивных исследований и получение новых результатов в данном направлении позволит продвинуться в решении указанных проблем.

\section{6. Заключение}

В работе представлен обзор основных достижений в области висмутовых волоконных лазеров и усилителей. В частности показано, что висмутовые волоконные лазеры перекрывают O, E, S, C, L и U телекоммуникационные полосы. Полученные результаты свидетельствуют о больших потенциальных возможностях использования висмутовых волоконных световодов для создания лазеров и оптических усилителей, работающих в ближнем ИК-диапазоне.

\section{Список литературы}

1. Morioka T. New generation optical infrastructure technologies: "EXAT 'initiative' towards 2020 and beyond" // OptoElectronics and Communications Conference. - 2009. - Paper FT4.

2. Дианов Е.М. На пороге пета-эры // Успехи физических наук. 2013. - T. 183, № 5. - С. 511-518.

3. Four extended-reach TDM PONs sharing a bidirectional hybrid CWDM amplifier / P.P. Iannone, H.H. Lee, K.C. Reichmann, X. Zhou, M. Du, B. Palsdottir, K. Feder, P. Westbrook, K. Brar, J. Mann, L. Spiekman // J. Lightwave Technol. - 2008. - Vol. 26. - P. 138-143.

4. Engineering an extended gain bandwidth hybrid Raman - optical Parametric amplifier for next generation CWDM PON / S. Peris, N. Madamopoulos, N. Antoniades, D. Richards, M.A. Ummy, R. Dirsinville // J. Lightwave Technol. - 2014. - Vol. 32, iss. 5. - P. 939-946.

5. Fujimoto Y., Nakatsuka M. Infrared Luminescence from BismuthDoped Silica Glass // Jpn. J. Appl. Phys. - 2011. - Vol. 40. - P. L279-L281.

6. Absorption, Fluorescence and Optical Amplification in MCVD Bismuth-Doped Silica Glass Optical Fibres / V.V. Dvoyrin, V.M. Mashinsky, E.M. Dianov, A.A. Umnikov, M.V. Yashkov, A.N. Guryanov // Proc. European Conf. on Optical Communications, September 25-29. - Glasgow, 2005. - Paper Th 3.3.5.

7. Silica-Based Bismuth-Doped Fiber for Ultra Broad Band LightSource and Optical Amplification around $1.1 \mu \mathrm{m} / \mathrm{T}$. Haruna, M. Kakui, T. Taru, Sh. Ishikawa, M. Onishi // Proc. Optical Amplifiers and Their Applications Topical Meeting, August 7-10. - Budapest, 2005. - Paper MC3. 
8. Dianov E.M. Bismuth-doped optical fibers: a challenging active medium for near-IR lasers and optical amplifiers // Light: Science and Applications 1. - 2012. - e 12. DOI: 10.1038/lsa. 2012.12.

9. Dianov E.M. Amplification in extended transmission bands using $\mathrm{Bi}-$ doped optical fibers // J. Lightwave Technol. - 2013. - Vol. 31. - P. 681-688.

10. Combined excitation-emission spectroscopy of bismuth active centers in optical fibers / S.V. Firstov, V.F. Khopin, I.A. Bufetov, E.G. Firstova, A.N. Guryanov, E.M. Dianov // Opt. Express. - 2011. - Vol. 19. P. 19551-19561.

11. Bufetov I.A., Dianov E.M. Bi-doped fiber lasers // Laser Phys. Lett. - 2009. - Vol. 6. - P. 487-504.

12. Непрерывный висмутовый волоконный лазер / Е.М. Дианов, В.В. Двойрин, В.М. Машинский, А.А. Умников, М.В. Яшков, А.Н. Гурьянов // Квантовая электроника. - 2005. - № 35 (12). - С. 1083-1084.

13. Bi-Doped Optical Fibers and Fiber Lasers / I.A. Bufetov, M.A. Melkumov, S.V. Firstov, K.E. Riumkin, A.V. Shubin, V.F. Khopin, A.N. Guryanov, E.M. Dianov // IEEE J. Selected Topics in Quantum Electronics. 2014. - Vol. 20. - P. 111-125.

14. Dvoyrin V.V., Mashinsky V.M., Dianov E.M. Efficient BismuthDoped Fiber Lasers // IEEE JOURNAL OF QUANTUM ELECTRONICS. 2008. - Vol. 44 (9). - P. 834-840.

15. / Efficient Bi-doped fiber lasers and amplifiers for the spectral region 1300-1500 nm / I.A. Bufetov, M.A. Melkumov, V.F. Khopin, S.V. Firstov, A.V. Shubin, O.I. Medvedkov, A.N. Guryanov, E.M. Dianov // Proc. of SPIE. - 2010. - Vol. 7580. - P. 758014-758023.

16. High-power $\mathrm{cw} 1.27 \mu \mathrm{m}$ Bi-doped fiber laser / I.A. Bufetov, A.V. Shubin, S.V. Firstov, M.A. Melkumov, V.F. Khopin, A.N. Guryanov, E.M. Dianov // Conf. on Lasers and Electro-optics (CLEO/Europe 2011). Munich, 2011. - Paper CJ8.2THU.

17. Bismuth-doped silica-based fiber lasers operating between 1389 and $1538 \mathrm{~nm}$ with output power of up to $22 \mathrm{~W} / \mathrm{A}$.V. Shubin, I.A. Bufetov, M.A. Melkumov, S.V. Firstov, O.I. Medvedkov, V.F. Khopin, A.N. Guryanov, E.M. Dianov // Optics Lett. - 2012. - Vol. 37. - P. 2589-2591.

18. Новый висмутовый волоконный лазер, излучающий в диапазоне 1625-1775 нм / Е.М. Дианов, С.В. Фирстов, С.В. Алышев, К.Е. Рюмкин, А.В. Шубин, В.Ф. Хопин, А.Н. Гурьянов, О.И. Медведков, М.А. Мелькумов // Квантовая электроника. - 2014. - Т. 44, № 6. C. 503-504. 
19. New Bismuth-Doped Fiber Laser Operating at 1625-1775 nm / E.M. Dianov, S.V. Firstov, S.V. Alyshev, K.E. Riumkin, S.V. Shubin, V.F. Khopin, A.N. Guryanov, O.I. Medvedkov, M.A. Melkumov // Proc. European Conf. on Optical Communications. - Cannes, 2014. - Paper number R1.5.

20. Laser diode pumped bismuth-doped optical fiber amplifier for $1430 \mathrm{~nm}$ band / M.A. Melkumov, I.A. Bufetov, A.V. Shubin, S.V. Firstov, V.F. Khopin, A.N. Guryanov, E.M. Dianov // Optics Lett. - 2011. - Vol. 36, no. 13. - P. 2408-2410.

21. Excited state absorption in various Bi-doped fibers / K.E. Riumkin, M.A. Melkumov, I.A. Varfolomeev, A.V. Shubin, I.A. Bufetov, S.V. Firstov, V.F. Khopin, A.A. Umnikov, A.N. Guryanov, E.M. Dianov // Optics Lett. -2014. - Vol. 39. - P. 2503-2506.

22. Bismuth-doped-glass optical fibers-a new active medium for lasers and amplifiers / V.V. Dvoyrin, V.M. Mashinsky, L.I. Bulatov, I.A. Bufetov, A.V. Shubin, M.A. Melkumov, E.F. Kustov, A.A. Umnikov, V.F. Khopin, M.V. Yashkov, A.N. Guryanov, E.M. Dianov // Optics lett. - 2006. - Vol. 31, no. 20. - P. 2966-2968.

23. ИК люминесценция в легированных висмутом германатных стеклах и волоконных световодах / А.А. Пыненков, С.В. Фирстов, А.А. Панов, Е.Г. Фирстова, К.Н. Нищев, И.А. Буфетов, Е.М. Дианов // Квантовая электроника. - 2013. - № 43 (2). - С. 174-176.

24. Influence of the melting conditions of heavy metal oxide glasses containing bismuth oxide on their optical absorption / O. Sanz, E. HaroPoniatowski, J. Gonzalo, J.M. Fernández Navarro // J. of Non-Crystalline Solids. - 2006. - Vol. 352, iss. 8. - P. 761-768.

\section{References}

1. Morioka T. New generation optical infrastructure technologies: "EXAT 'initiative' towards 2020 and beyond". OptoElectronics and Communications Conference, 2009, paper FT4.

2. Dianov E.M. Na poroge peta-ery [On the threshold of the peta-era]. Uspekhi fizicheskikh nauk, 2013, vol. 183, no. 5, pp. 511-518.

3. Iannone P.P, Lee H.H., Reichmann K.C., Zhou X., Du M., Palsdottir B, Feder K., Westbrook P., Brar K., Mann J., Spiekman L. Four extended-reach TDM PONs sharing a bidirectional hybrid CWDM amplifier. J. Lightwave Technol, 2008, vol. 26, pp. 138-143.

4. Peris S., Madamopoulos N., Antoniades N., Richards D., Ummy M.A., Dirsinville R. Engineering an extended gain bandwidth hybrid Raman - optical 
Parametric amplifier for next generation CWDM PON. J. Lightwave Technol, 2014, vol. 32, iss. 5, pp. 939-946.

5. Fujimoto Y., Nakatsuka M. Infrared Luminescence from BismuthDoped Silica Glass. Jpn. J. Appl. Phys., 2001, vol. 40, pp. L279-L281.

6. Dvoyrin V.V., Mashinsky V.M., Dianov E.M., Umnikov A.A., Yashkov M.V., Guryanov A.N. Absorption, Fluorescence and Optical Amplification in MCVD Bismuth-Doped Silica Glass Optical Fibres. Proc. European Conf. on Optical Communications. Glasgow, 2005, paper Th 3.3.5.

7. Haruna T., Kakui M., Taru T., Ishikawa Sh., Onishi M. Silica-Based Bismuth-Doped Fiber for Ultra Broad Band Light-Source and Optical Amplification around 1.1 $\mu \mathrm{m}$. Proc. Optical Amplifiers and Their Applications Topical Meeting. Budapest, 2005, paper MC3.

8. Dianov E.M. Bismuth-doped optical fibers: a challenging active medium for near-IR lasers and optical amplifiers. Light: Science and Applications 1, 2012, e 12. DOI: 10.1038/lsa. 2012.12.

9. Dianov E.M. Amplification in extended transmission bands using Bidoped optical fibers. J. Lightwave Technol, 2013, vol. 31, pp. 681-688.

10. Firstov S.V., Khopin V.F., Bufetov I.A., Firstova E.G., Guryanov A.N. and Dianov E.M. Combined excitation-emission spectroscopy of bismuth active centers in optical fibers. Opt. Express, 2011, vol. 19, pp. 19551-19561.

11. Bufetov I.A., Dianov E.M. Bi-doped fiber lasers. Laser Phys. Lett., 2009, vol. 6, pp. 487-504.

12. Dianov E.M., Dvoirin V.V., Mashinskii V.M., Umnikov A.A., Iashkov M.V., Gur'ianov A.N. Nepreryvnyi vismutovyi volokonnyi lazer [CW bismuth fibre laser]. Kvantovaia elektronika, 2005, no. 35 (12), pp. 1083-1084.

13. Bufetov I.A., Melkumov M.A., Firstov S.V., Riumkin K.E., Shubin A.V., Khopin V.F., Guryanov A.N., Dianov E.M. Bi-Doped Optical Fibers and Fiber Lasers IEEE J. Selected Topics in Quantum Electronics, 2014, vol. 20, pp. 111-125.

14. Dvoyrin V.V., Mashinsky V.M., Dianov E.M. Efficient BismuthDoped Fiber Lasers. IEEE JOURNAL OF QUANTUM ELECTRONICS, 2008, vol. 44 (9), pp. 834-840.

15. Bufetov I.A., Melkumov M.A., Khopin V.F., Firstov S.V., Shubin A.V., Medvedkov O.I., Guryanov A.N., Dianov E.M. Efficient Bi-doped fiber lasers and amplifiers for the spectral region 1300-1500 nm. Proc. of SPIE, 2010, vol. 7580, pp. 758014-758023.

16. Bufetov I.A., Shubin A.V., Firstov S.V., Melkumov M.A., Khopin V.F., Guryanov A.N., Dianov E.M. High-power cw $1.27 \mu \mathrm{m}$ Bi-doped fiber laser. Conf. 
on Lasers and Electro-optics (CLEO/Europe 2011). Munich, 2011, paper CJ8.2THU.

17. Shubin A.V., Bufetov I.A., Melkumov M.A., Firstov S.V., Medvedkov O.I., Khopin V.F., Guryanov A.N., Dianov E.M. Bismuth-doped silicabased fiber lasers operating between 1389 and $1538 \mathrm{~nm}$ with output power of up to 22 W . Optics Lett., 2012, vol. 37, pp. 2589-2591.

18. Dianov E.M., Firstov S.V., Alyshev S.V., Riumkin K.E., Shubin A.V., Khopin V.F., Gur'ianov A.N., Medvedkov O.I., Mel'kumov M.A. Novyi vismutovyi volokonnyi lazer, izluchaiushchii v diapazone 1625-1775 nm [A new bismuth-doped fibre laser, emitting in the range 1625-1775 nm]. Kvantovaia elektronika, 2014, vol. 44, no. 6, pp. 503-504.

19. Dianov E.M., Firstov S.V., Alyshev S.V., Riumkin K.E., Shubin S.V., Khopin V.F., Guryanov A.N., Medvedkov O.I., Melkumov M.A. New BismuthDoped Fiber Laser Operating at 1625-1775 nm. Proc. European Conf. on Optical Communications. Cannes, 2014, paper number R1.5.

20. Melkumov M.A., Bufetov I.A., Shubin A.V., Firstov S.V., Khopin V.F., Guryanov A.N., Dianov E.M. Laser diode pumped bismuthdoped optical fiber amplifier for $1430 \mathrm{~nm}$ band. Optics Lett., 2011, vol. 36, no. 13, pp. 2408-2410.

21. Riumkin K.E., Melkumov M.A., Varfolomeev I.A., Shubin A.V., Bufetov I.A., Firstov S.V., Khopin V.F., Umnikov A.A., Guryanov A.N., Dianov E.M. Excited state absorption in various Bi-doped fibers. Optics Lett., 2014, vol. 39, pp. 2503-2506.

22. Dvoyrin V.V., Mashinsky V.M., Bulatov L.I., Bufetov I.A., Shubin A.V., Melkumov M.A., Kustov E.F., Umnikov A.A., Khopin V.F., Yashkov M.V., Guryanov A.N., Dianov E.M. Bismuth-doped-glass optical fibers-a new active medium for lasers and amplifiers Optics lett., 2006, vol. 31, no. 20, pp. 2966-2968.

23. Pynenkov A.A., Firstov S.V., Panov A.A., Firstova E.G., Nishchev K.N., Bufetov I.A., Dianov E.M. IK liuminestsentsiia v legirovannykh vismutom germanatnykh steklakh i volokonnykh svetovodakh [IR luminescence in bismuth-doped germanate glasses and fibres]. Kvantovaia elektronika, 2013, no. 43 (2), pp. 174-176.

24. Sanz O., Haro-Poniatowski E., Gonzalo J., Fernández Navarro J.M. Influence of the melting conditions of heavy metal oxide glasses containing bismuth oxide on their optical absorption J. of Non-Crystalline Solids, 2006, vol. 352, iss. 8, pp. 761-768.

Получено 15.10.2014 